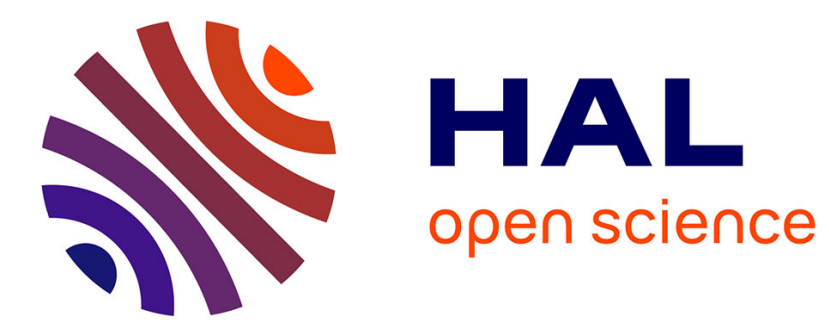

\title{
Linear Elastic Properties of the Facial Soft Tissues Using an Aspiration Device: Towards Patient Specific Characterization.
}

\author{
Vincent Luboz, Emmanuel Promayon, Yohan Payan
}

\section{To cite this version:}

Vincent Luboz, Emmanuel Promayon, Yohan Payan. Linear Elastic Properties of the Facial Soft Tissues Using an Aspiration Device: Towards Patient Specific Characterization.. Annals of Biomedical Engineering, 2014, epub ahead of print. 10.1007/s10439-014-1098-1 . hal-01061677

\section{HAL Id: hal-01061677 https://hal.science/hal-01061677}

Submitted on 8 Sep 2014

HAL is a multi-disciplinary open access archive for the deposit and dissemination of scientific research documents, whether they are published or not. The documents may come from teaching and research institutions in France or abroad, or from public or private research centers.
L'archive ouverte pluridisciplinaire HAL, est destinée au dépôt et à la diffusion de documents scientifiques de niveau recherche, publiés ou non, émanant des établissements d'enseignement et de recherche français ou étrangers, des laboratoires publics ou privés. 


\section{Linear elastic properties of the facial soft tissues using an aspiration device:}

$4 \quad$ V. Luboz ${ }^{1}$, E. Promayon ${ }^{1}$, Y. Payan ${ }^{1} *$

51 UJF-Grenoble1/CNRS/TIMC-IMAG UMR 5525, Grenoble, F-38041, France, \{vincent.luboz, 6 Emmanuel.Promayon, ypayan\}@imag.fr;

7

8

9

20

Abbreviated title: Characterization of soft tissues elastic properties

*Corresponding author:

Yohan Payan

Equipe GMCAO - Laboratoire TIMC-IMAG

Université Joseph Fourier - CNRS UMR 5525

Pavillon Taillefer

Faculté de Médecine - 38706 La Tronche cedex - France

Tel: +33 (0)4 56520001 - Fax: +33 (0)4 56520055

email: Yohan.Payan@imag.fr

To appear in Annals of Biomedical Engineering, DOI: 10.1007/s10439-014-1098-1. 


\section{Abstract}

23 Biomechanical modeling of the facial soft tissue behavior is needed in aesthetic or maxillofacial surgeries where the simulation of the bone displacements cannot accurately predict the visible outcome on the patient's face. Because these tissues have different nature and elastic properties across the face, depending on their thickness, and their content in fat or muscle, individualizing their mechanical parameters could increase the simulation accuracy. Using a specifically designed aspiration device, the facial soft tissues deformation is measured at four different locations (cheek, cheekbone, forehead, and lower lip) on 16 young subjects. The stiffness is estimated from the deformations generated by a set of negative pressures using an inverse analysis based on a Neo Hookean model. The initial Young's modulus of the cheek, cheekbone, forehead, and lower lip are respectively estimated to be $31.0 \mathrm{kPa} \pm 4.6,34.9 \mathrm{kPa} \pm$ 6.6, 17.3 $\mathrm{kPa} \pm 4.1$, and $33.7 \mathrm{kPa} \pm$ 7.3. Significant intra-subject differences in tissue stiffness are highlighted by these estimations. They also show important inter-subject variability for some locations even when mean stiffness values show no statistical difference. This study stresses the importance of using a measurement device capable of evaluating the patient specific tissue stiffness during an intervention. 


\section{Introduction}

43

In aesthetic and maxillo-facial surgery, most of the interventions are related to the bony structures and aim either at repairing functionalities of the oro-facial structures or improving their shape. To predict the final shape of the face after the displacement of the bones, most of the surgeons rely on their experience. Nevertheless, because of anatomical variations in the facial soft tissues, i.e., in the amount of fat between the muscles, the thickness of the skin, or their material parameters, predictions may not always be accurate. Several research groups have presented simulators to help in the evaluation of the outcomes of aesthetic and/or maxillo-facial surgeries. For example, Chabanas et al. ${ }^{6}$ presented a Finite Element (FE) model of the skull and face used as an atlas which can be deformed to fit the patient's anatomy. In this study, the soft tissues were modeled as a homogeneous, linear elastic material and its material parameters were chosen to fit a clinical case where pre- and post-operative CT scans were available. It led to a Young's modulus of $15 \mathrm{kPa}$, and a Poisson's ratio $v$ of 0.49 . Other finite element models also based on a linear modeling of the soft tissues have been presented in the literature ${ }^{3,12,14,22}$. In these articles, the mechanical parameters were chosen either by comparing their simulation results with imaging data acquired from patients or were values from the soft tissue literature, although not specifically measured on the facial soft tissues. The validation of the predictions given by these simulators were therefore complicated by the fact that these mechanical parameters were either only representative of a single patient, or were not directly related to the facial soft tissues, or were 
extracted from ex vivo measurements (and therefore might be different from in vivo because of the lack of perfusion and the difference of temperature ${ }^{13}$ ).

To improve the evaluation of these FE models, it seems important to be able to determine the material parameters of the facial soft tissues for a specific patient, or at least to give an accurate cartography of the face mechanical properties for an average patient, if possible. In a first attempt to reach this aim, an aspiration device, called the Cutometer (http://www.courage-khazaka.de $)^{7,18}$, was used to define several specific parameters such as immediate distension, delayed distension, immediate retraction, or final deformation of the face skin. These studies showed that viscoelastic properties are significantly influenced by aging. Unfortunately, in both studies, no clear link can be established between the Cutometer specific parameters and the more classical parameters used in mechanical constitutive laws. This device is mainly aimed at helping dermatologists in their need to quantify the elasticity in an aging trend more than for mechanical simulation purpose. Sonographic elastography has proven its ability to estimate the stiffness of soft tissues for maxillo-facial applications ${ }^{2}$. It provides a map of Young moduli for tissues' superficial layers which values can only be used for simulations assuming a small deformations framework. Other studies ${ }^{4,5,9,15,17}$ tried to mechanically quantify the skin stiffness either by direct measurements or by using inverse methods to match deformations visualized on medical images. Unfortunately the initial Young's modulus (i.e., the initial slope of the stress-strain curve) and Poisson's ratio reported in these studies had fairly important ranges. For example, the Young's modulus was estimated by Bickel et al. ${ }^{5}$ to $78 \mathrm{kPa}$ and the Poisson's ratio to 0.47 using a Neo Hookean model. The Young's modulus was found to vary between $4 \mathrm{kPa}$ and $18.8 \mathrm{MPa}$ in in vivo and in vitro measurements according to Lapeer et al. ${ }^{15}$. Another study ${ }^{17}$ also evaluated the initial Young's 
modulus to $15 \mathrm{kPa}$ using a Mooney Rivlin model (where $\mathrm{C}_{10}=2.5 \mathrm{kPa}$ ). In a previous study ${ }^{9}$, we evaluated the in vitro stiffness of the cheek of a fresh cadaver to $15 \mathrm{kPa}$. This work was done in the context of a maxillo-facial simulator. The skin initial Young's modulus was evaluated to $22.8 \mathrm{kPa}$ using a Mooney Rivlin model (where $\mathrm{C}_{10}=3.8 \mathrm{kPa}$ ) and measurements on different locations on the face of one subject by Barbarino et al. ${ }^{4}$ In a recent study, a micro-robotic device was used by Flynn et al. ${ }^{8}$ to record the force-displacement response of the cheek of five volunteers. Facial skin exhibits a non-linear, anisotropic, and viscoelastic force-displacement response. When modeled using an Ogden FE model, the skin initial Young's modulus was found to be between $15.9 \mathrm{kPa}$ and $89.4 \mathrm{kPa}$. Flynn et al. ${ }^{8}$ also showed variations in stiffness between different locations on the face for one subject. Such a paper stems questions about patient specific variations of the stiffness of the facial soft tissues and the stiffness variation depending on the location for different subjects.

In the present paper, we aim at clarifying these patient specific variations in order to improve the planning of different maxillo-facial surgeries using FE model. The objective is to provide a tool to evaluate the facial soft tissue stiffness (characterized here with the Young's modulus) while being compatible with the constraints of the operating room. The goal is to evaluate the in vivo initial Young's modulus for the facial soft tissues at four representative locations, namely the cheek, the cheekbone, the forehead, and the lower lip, and for a panel of subjects of different ages and body mass indexes. For the maxillo-facial application, a quasistatic framework can be assumed since boundary conditions corresponding to bone displacements are applied and simulated without taking into account the dynamic viscoelastic behavior of the soft tissues. 


\section{Materials and Methods}

A device characterizing the mechanical behavior of the soft tissues was used to determine their initial Young's modulus. This device, called LASTIC (for Light Aspiration device for in vivo Soft TIssue Characterization), is based on the aspiration technique and was

first introduced by Schiavone et al. ${ }^{19}$ while quantifying the brain behavior ${ }^{20}$. This technique

117 has also been used by Hollenstein et al. ${ }^{11}$ for other applications such as the uterus or the liver.

118 It also has been used on various in vivo tissues such as the forearm skin and the tongue ${ }^{21}$. In its current version, Figure 1, LASTIC is a $33 \mathrm{~mm}$ x $34 \mathrm{~mm}$ metal cylinder composed of two compartments. The lower one is an airtight chamber, open at the bottom by a $12 \mathrm{~mm}$ diameter circular aperture and closed at the top by a glass window. The upper compartment holds the electronic part consisting of a miniature 2 megapixel digital camera and a LED used as a light source. The aspiration chamber is connected to a programmable syringe pump that can generate a negative pressure (measured by a manometer) which deforms the tissues on which LASTIC is laid on. This deformation is imaged by the camera via a 45 degree inclined mirror which provides a view of the tissue from the side. The height of the tissue deformation is segmented on the recorded image. A basic camera calibration is performed to determine the pixel size. On average, the pixel size is around $0.01 \mathrm{~mm}$. Measuring the deformation height corresponding to several steps of increasing negative pressures can give an estimation of the behavior of the tissues. LASTIC is fully sterilizable and can consequently be used inside the sterile fields of operating rooms. 
The tissue measurements are then processed through an inverse analysis to estimate

133 the tissue mechanical behavior. This analysis consists of matching the measured

134 deformation/pressure curve to a pre-computed library of displacement heights determined by

135 a FE Analysis of the aspiration experiment using a Neo-Hookean constitutive law ${ }^{1}$. Such a

136 material is fairly stable and shows a behavior similar to the one simulated by Yeoh or

137 Mooney-Rivlin materials at strains levels observed for such aspiration experiments. The Neo-

138 Hookean equation is written as:

$139 \mathrm{~W}=\mathrm{C}_{10}\left(\mathrm{I}_{1}-3\right)+(\mathrm{J}-1)^{2} / \mathrm{D}$

140 where $I_{1}$ is the first invariant of the left Cauchy-Green deformation tensor, $C_{10}$ is a material 141 parameter, $\mathbf{J}$ is the determinant of the deformation gradient $\mathrm{F}, \mathrm{D}$ is a material 142 incompressibility parameter (with $\mathrm{D}=(1-2 \mathrm{v}) / \mathrm{C}_{10}$ ), and $\mathrm{W}$ is the strain energy. As in Chabanas 143 et al. ${ }^{6}$, the skin is assumed to be nearly incompressible and a Poisson's ratio $v$ of 0.49 was 144 chosen. Note that, for small extensions (i.e., in the linear elastic domain when $\mathrm{I}_{1}$ is close to 3 ) 145 the initial Young's modulus, i.e., the initial slope of the stress-strain curve, can be 146 approximated by $\mathrm{E}=6 \mathrm{C}_{10}$.

147 The facial soft tissues are modeled by a thick circular slice while LASTIC is described 148 by a rigid hollow cylinder (Figure 2). Taking advantage of the axisymmetric geometry of our 149 model, the mechanical study is reduced to a two-dimensional structural analysis. The sample 150 is meshed with approximately 2,000 linear quad elements. The mesh is refined in the 151 neighborhood of the aspirated region, where highest deformation occurs, in order to increase 152 the accuracy of the computed solution. The interface between LASTIC and the sample is 153 specifically meshed with contact elements in order to ensure that the aspirated skin tissue 154 slides without friction inside the LASTIC hole. 
Given the different thicknesses of the studied facial tissues, an estimation of their variation was performed on a CT scan of a head (courtesy of subject \#4). It presented a thickness of 5 to $6 \mathrm{~mm}$ for the forehead skin and of $15 \mathrm{~mm}$ or more for the tissues of the lip, cheek and cheekbone. To take this variation into account, two different axisymmetric models were used: one with a tissue thickness of $5 \mathrm{~mm}$ and one with a thickness of $15 \mathrm{~mm}$ (Figure 2). There was no need to create a model for each location over $15 \mathrm{~mm}$ because the maximal negative pressure created by LASTIC does not influence layers over $13 \mathrm{~mm}$, see Figure 2. On the other hand, for the model with a tissue thickness of $5 \mathrm{~mm}$, the maximal negative pressure created by LASTIC leads to a deformation of the tissues that is influenced by the tissue thickness. In this case, the maximum deformation measured in the tissues is around $13 \%$ (assuming that we do not consider the specific region of contacts between the tissues and the LASTIC cylinder). For a tissue thickness of $15 \mathrm{~mm}$, the maximum deformation is $17 \%$. These two values show that the material deformations are not too large; using a Neo Hookean model (thus neglecting the non-linearity due to very large deformations) is therefore relevant here.

The pre-computed library of displacements was generated using a wide range of $\mathrm{C}_{10}$ and applied negative pressure for the two different thicknesses. Matching the measurements with the corresponding library (the $5 \mathrm{~mm}$ model for the forehead and the $15 \mathrm{~mm}$ model for the lip, cheek, and cheekbone), using a least-square minimization method, leads to an estimation of the $\mathrm{C}_{10}$ value corresponding to the tissue stiffness. This minimization is performed in less than a second.

The device has been validated in Luboz et al. ${ }^{16}$ on several types of samples including silicone rubbers, with stiffness ranging from $10 \mathrm{kPa}$ to $90 \mathrm{kPa}$, and compared to tensile tests. 
178 This validation showed that LASTIC overestimates the stiffness by $16 \%$ on average with a 179 standard deviation of $9.5 \%$. This overestimation is mainly due to errors generated during the 180 acquisition, namely due to the manometer precision and the camera calibration, which decreases the precision of the image segmentation to evaluate the tissue deformation.

2.2 Cartography of the face stiffness locations with presumably different tissue thicknesses and different amounts of fat and muscle were consequently chosen: the cheek, the cheekbone, the forehead, and the lower lip. To 
location varied for each subject. The initial negative pressure was $0 \mathrm{kPa}$ while the maximal

201 negative pressure was up to $7.3 \mathrm{kPa}$ (=73 mbar), depending on the subject and location.

202 To avoid any leaks at the interface between LASTIC and the tissues, a water wet 203 gauze compress was used to wipe the skin before each measurement. This set up left a slight 204 amount of water facilitating the suction and reducing the viscosity. Furthermore, LASTIC was 205 very slightly pressed on the tissue by the subjects to ensure that the bottom compartment 206 entire surface lies on the skin. The first three measured values were not used during the 207 minimization process in order to compensate the initial load applied by the positioning of LASTIC, which could be observed as a bump on the video screen. For each location, five measurements were made repeatedly: the first measurement was performed successively on the cheek, the cheekbone, the forehead, and the lower lip; then, the second measurement was performed successively on the cheek, the cheekbone, the 212 forehead, and the lower lip, etc... until the fifth measurements was performed the same way. 213 This repetitive cycle allowed the tissue of each location to relax between each measurement. 214 A single measurement took about three minutes which means that overall, each subject's 20 measurements were performed in about one hour.

216 During all the measurements, the subjects were asked to stay as relaxed as possible in 217 order to keep the activations of the underlying muscles of the face as low as possible and consequently to reduce the impact of tissue anisotropy and initial tension on the 219 measurements.

\section{Results}


The stiffnesses of the facial soft tissues (characterized here with the initial Young's

modulus) measured with LASTIC on 16 healthy voluntary subjects at four locations, as well as the sex, age and BMI of each subject, are shown in Table 1. The cheek, cheekbone, forehead, and lower lip mean stiffnesses of each subject, as well as the standard deviation resulting from the five measurements at each location, are given in the four last columns. The overall subjects' mean stiffness for each of the four locations is also given on the bottom row. The corresponding initial Young's modulus are $\mathrm{E}_{\mathrm{c}}=31.0 \mathrm{kPa} \pm 4.6$ for the cheek, $\mathrm{E}_{\mathrm{cb}}=34.9$ $\mathrm{kPa} \pm 6.6$ for the cheekbone, $\mathrm{E}_{\mathrm{f}}=17.3 \mathrm{kPa} \pm 4.1$ for the forehead, and $\mathrm{E}_{1}=33.7 \mathrm{kPa} \pm 7.3$ for the lower lip. Neither tissue stiffening nor softening is observed for the repeated measurements; we therefore assume that there is probably little influence of the pre conditioning.

The complete measurements are given in the supplementary material. Figure 4 presents a Whisker box plot showing the mean, minimal and maximal initial Young's modulus (in $\mathrm{kPa}$ ) for the cheek, cheekbone, forehead and lower lip. The result of the bilateral paired Welch's T-test between the forehead and the three other locations is also presented. This test is an adaptation of the Student's t-test intended for use with two samples having possibly unequal variances ${ }^{23}$.

Table 2 shows the results of a bilateral paired Welch's T-test between each measurement location. The p-value resulting from this test demonstrates that the difference between the mean stiffness of the forehead $E_{f}$ and the cheek $E_{c}(p=2.5 E-5)$ is statistically significant $(p<=0.05)$. This is also significant for the difference between $E_{f}$ and $E_{1}(p=7 E-6)$ and for $E_{f}$ and $E_{c b}(p=1 E-6)$. On the other hand, there are no statistical differences between $E_{c}$ and $\mathrm{E}_{\mathrm{l}}$, between $\mathrm{E}_{\mathrm{c}}$ and $\mathrm{E}_{\mathrm{ch}}$, and between $\mathrm{E}_{\mathrm{l}}$ and $\mathrm{E}_{\mathrm{ch}}$. 
It is to be noted that the evaluated mean stiffnesses do not show any dependence

247 neither with the age, BMI or sex.

248 Nevertheless, subject to subject differences can be observed for each location even if 249 there is no overall statistical difference. It is the case for example for the cheekbone and lower lip stiffness of subjects \#5 and \#15: $E_{c b}(5)=22.1 \mathrm{kPa}$ and $E_{1}(5)=33.3 \mathrm{kPa}$ while

$\mathrm{E}_{\mathrm{cb}}(15)=35.1 \mathrm{kPa}$ and $\mathrm{E}_{\mathrm{l}}(15)=22.3 \mathrm{kPa}$. Another example of the disparity can be seen between subjects \#10 and \#12 for the cheek and lower lip stiffness: $E_{c}(10)=37.1 \mathrm{kPa}$ and $\mathrm{E}_{1}(10)=28.0 \mathrm{kPa}$ while $\mathrm{E}_{\mathrm{c}}(12)=31.6 \mathrm{kPa}$ and $\mathrm{E}_{\mathrm{l}}(12)=45.7 \mathrm{kPa}$. These two subjects have completely different stiffness values compared to the mean stiffness $\mathrm{E}_{1}$ for the lower lip (it can be observed that $E_{1}(10)=E_{1} \times 83 \%$ while $E_{1}(12)=E_{1} \times 135 \%$.), see Figure 5, and for the cheek while overall there is no statistical difference between these two locations $(\mathrm{p}=0.424)$. For all these values, the standard deviations for the five measurements used to obtain these mean values are relatively low (between 4.0 and $7.9 \mathrm{kPa}$ ).

\section{Discussion}

The stiffness values presented in Table 1 and Figure 4 and outlined in the previous paragraph show fairly important variations between subjects, even if there is no statistical difference over the whole subject pool. The cheek stiffness measured on all the 16 subjects fall within the range given by Flynn et al. ${ }^{8}$ (which is based on cheek measurements on five volunteers): from $15.9 \mathrm{kPa}$ to $89.4 \mathrm{kPa}$.

As shown in Table 2, the stiffnesses of the forehead and the cheek, of the forehead and the lower lip, and of the forehead and the cheekbone are statistically different. It can be 
explained by the smaller tissue thickness (and different boundary conditions) for the forehead as compared to the cheek, the lower lip, and the cheekbone. Precisely measuring tissue thicknesses at different locations (with MRI or US imaging) should help in understanding more deeply these differences Measuring and estimating variations in tissue types or muscles activations should also provide some clues to explain the observed differences.

A first estimation of facial tissue stiffness can nonetheless be given with our LASTIC measurements. For a given location, the inter-subject variability can be estimated by the standard deviation $\operatorname{sd}\left(\mathrm{E}_{\mathrm{i}} / \mathrm{E}\right)$, where $\mathrm{E}_{\mathrm{i}}$ is the normalized Young moduli of subject $\mathrm{i}$ and $\mathrm{E}$ is the average Young modulus of all $\mathrm{E}_{\mathrm{i}}$. The inter-subject variability $\operatorname{sd}\left(\mathrm{E}_{\mathrm{i}} / \mathrm{E}\right)$ is equal to $27 \%, 29$ $\%, 29 \%$, and $27 \%$, respectively for the forehead, cheek, lower lip, and cheekbone. For a given location, the intra-subject variability can be estimated by the average standard deviation mean $\left(\operatorname{sd}\left(E_{i, j} / E_{i}\right)\right)$, where $E_{i, j}$ is the resulting measured Young modulus for the measurement $j$ of subject $\mathrm{i}$. The intra-subject variability mean $\left(\mathrm{sd}\left(\mathrm{E}_{\mathrm{i}, \mathrm{j}} / \mathrm{E}_{\mathrm{i}}\right)\right)$ is equal to $22 \%, 16 \%, 21 \%, 19 \%$, respectively for the forehead, cheek, lower lip, and cheekbone. This shows that for a given location, the intra-subject variability (i.e., the standard deviation per subject) is smaller than the inter-subject variability (i.e., the standard deviation per location). Consequently the small variation of position due to the repositioning of the device between two measurements for the same location has less effect on the estimation than the change of stiffness from a subject to another. Furthermore, we could question the fact that muscle activation could play a role in the estimation of the stiffness since two locations include muscles that can be voluntarily activated (i.e., lip and forehead) while the other two include muscles that are more difficult to activate voluntarily (i.e., cheek and cheekbone). The lower lip and forehead have indeed higher inter-subject variability than the cheek and cheekbone. Finally, it is important to note 
that the maximal difference between location variability being $6 \%$ (between forehead and cheek), these variations could also be explained by a change of device positioning, or a measurement error (see our previous work ${ }^{15}$ for an estimation of LASTIC errors).

The stiffness differences pointed out in the last part of the results section illustrate the fact that even if there is no statistical difference over the whole subject pool between some locations, for example between cheekbone and lower lip, there are fairly important variations of the stiffness between subjects. For example, it would be inaccurate to take the mean value $E_{\mathrm{cb}}=34.9 \mathrm{kPa}$ for the cheekbone for subjects \#4 and \#13 while the measured stiffness values were evaluated to $\mathrm{E}_{\mathrm{cb}}(4)=50.7 \mathrm{kPa}$ and $\mathrm{E}_{\mathrm{cb}}(13)=51.6 \mathrm{kPa}$, which would correspond to an underestimation of $30 \%$. The same observation can be made for the stiffness of the lower lip in subject \#8: the mean value $E_{1}=33.7 \mathrm{kPa}$ while the subject's stiffness value was estimated to $\mathrm{E}_{\mathrm{l}}(8)=17.9 \mathrm{kPa}$; this would lead to an over-estimation of $88 \%$. It therefore seems essential in the context of FE model for surgical planning to take these inter-subject variations into account.

Two types of limitations should be considered for this study: the first one concerns the experimental set up, and the second one concerns the mechanical set up. The experimental set up has four main limitations: the fact that muscle activation is not recorded during the experiments, the assumption that the facial soft tissues are homogeneous, the assumption that those tissues exhibit a linear stress-strain response, and the low variation of the subject pool in terms of age and BMI. The main limitation is relative to muscle activation. Even though subjects were asked to be as relaxed as possible, we cannot guarantee that their muscles were not activated at all, which would consequently have biased the measurements by stiffening the tissues. Because this activation could not be measured without using invasive EMG sensors, it 
315 is likely that the measurements with high standard deviation are the consequence of 316 involuntary muscle activation.

317 Assuming that the facial soft tissues are homogeneous is also erroneous: they are 318 composed of several layers of skin (including epidermis, dermis and hypodermis), muscles 319 and fat and can be more than a centimeter thick. Given the level of aspiration generated by our LASTIC device (never higher than $7.3 \mathrm{kPa}$ and a maximum tissue bump of about $5.1 \mathrm{~mm}$, with a maximal pre-load of $3.2 \mathrm{~mm}$, leading to an actual deformation directly due to the 322 suction of $1.9 \mathrm{~mm}$ ), the obtained characterization is mainly limited to the superficial layers of the facial tissue (epidermis and dermis), few millimeters below the skin at most. Assuming that these tissues are homogeneous consequently results in a non-completely accurate stiffness estimation. The stiffness estimation could be improved by considering a heterogeneous model with several layers of tissues with different mechanical properties. For instance, a two layer model including a thin superficial layer for the skin and a thicker one for 328 the underlying tissues could be considered as a better approximation of the face tissues. 329 Measuring the facial soft tissue stiffness in their full thickness would also need a higher level of negative pressure, which would probably have been refused by the Ethics Committee because of the risk for generating pain or damaging the tissues.

Assuming that the facial soft tissues exhibit a linear stress-strain response is also inaccurate for large deformations: because of their heterogeneity, the tissues mainly have a nonlinear response ${ }^{9}$. Estimating the Young's modulus corresponding to a Neo Hookean constitutive law is consequently accurate only for the initial low strain stiffness. Using a more complex constitutive law, such as one derived from a Mooney Rivlin or Ogden formulation, would probably improve the accuracy of the stiffness estimation with LASTIC. 
Finally, despite some individual differences, the relatively small variations of the

stiffness measured on our group of subjects can be explained by its low variance in age and in BMI. The subjects being all young or relatively young (between 23 and 44) their skin is likely to be in good shape and fairly elastic. As for the subject's BMI, it ranges between 16.8 and 26, and only four subjects are outside the standard deviation range $[19.4 ; 23.6]$. Most of the subjects therefore have probably a small amount of fat tissues under their face skin. The studied group is consequently not extremely representative of the world population neither in age nor in BMI.

Three main limitations can be listed concerning the mechanical set up: the low level of negative pressure generated by LASTIC, the initial load that may have been applied on the soft tissues, and the possible inaccuracy or non-reproducibility of the position of the measurements on each subject.

As stated above, the level of aspiration generated by LASTIC is never higher than 7.3 $\mathrm{kPa}$ which therefore limits the estimation of the stiffness to only superficial tissues. Using higher negative pressures could help to quantify deeper tissues but could also injure the subject; this was consequently not performed in our study.

Another source of inaccuracy in the stiffness estimation is due to the fact that the initial load applied on the soft tissues at the beginning of each measurement (to avoid any leaks) is not simulated in the FE model nor used for the inverse analysis. Removing the first three measured values seems a reasonable approximation as it is assumed that the pressure compensates the initial load and the consequent deformation of the tissues ${ }^{15}$. The initial load is always kept below the precision threshold of the manometer by checking the measured pressure on the manometer and the camera image. The degree of this initial pressure applied 
by the subjects is controlled during and after the experiments. This initial load creates an initial deformation of the tissues and a light aspiration has consequently no influence on them. Once the pressure is strong enough to aspirate the tissues, the tissue deformation is visible on the camera image. This threshold was reached around the third pressure step. Nevertheless, this compensation is not accurate and might lead to a deviation of the stiffness value.

The inaccuracy of the location of the measurements for each subject could also be responsible for increasing the resulting variance. The experimenter visually estimates the positioning error to a maximum of $5 \mathrm{~mm}$; we therefore assume that this has a minimal effect on the stiffness evaluation.

\section{Conclusion}

A map of the stiffness of the facial soft tissues is presented in this paper as measured by LASTIC, a device based on the aspiration technique. Using an inverse analysis with a FE Neo Hookean behavior, it provides an estimation of the stiffness of the tissues at four locations: the cheek, the cheekbone, the forehead and the lower lip. On average, the stiffness coefficient of the soft tissues composing the cheek, the cheekbone, the forehead, and the lower lip are respectively estimated to be $31.0 \mathrm{kPa}, 34.9 \mathrm{kPa}, 17.3 \mathrm{kPa}$, and $33.7 \mathrm{kPa}$. Considering all measurements, it seems difficult to obtain and use values describing an average patient. With statistical differences between the forehead and the cheek $(p=2.5 \mathrm{E}-5)$, between the forehead and the lower lip ( $\mathrm{p}=7 \mathrm{E}-6)$ and between the forehead and the cheekbone $(\mathrm{p}=1 \mathrm{E}-6)$, this study shows that the tissue thickness as well as the amount and nature of fat tissues and muscles below the skin probably play a role in the stiffness. Although no 
dependences can be exhibited between the mean tissue stiffnesses and neither the age, the BMI nor the sex of the subjects, this study gives an interesting first insight in the variation of the stiffness between subjects at different locations on the human face. The inter-subject variations appearing in our measurements are pointing out that in vivo patient specific measurements are essential to accurately model the facial soft tissues and that an average stiffness value is not adequate for a patient specific model. Specifically designed small aspiration devices are a convenient and practical way for doing so. As LASTIC can be sterilized, it could be used routinely during clinical practice in order to assess rapidly the patient specific tissue stiffness during the planning of aesthetic or maxillo-facial surgeries.

Future works will aim at correcting the limitations of this study. Firstly, the range of the population tested with LASTIC should be broaden by measuring the stiffness of the tissues on younger and older subjects and with a larger variation in BMI. Secondly, the accuracy and reproducibility of the device positioning during the measurements could also be improved, for instance by marking precisely onto the skin the aspiration locations. Increasing the level of negative pressure that LASTIC can generate in order to quantify the stiffness of deeper tissues is also possible but can only be done after evaluating the risk of damaging the skin. Quantifying the properties of the different skin layers and underlying tissues could also be implemented by using different sizes of orifice for the suction similarly to the work of Hendricks et al. ${ }^{10}$. Another improvement to be addressed is the fact that following only the deformation of one point at the top of the tissue aspired dome is not enough to evaluate soft tissues' anisotropy. We therefore plan to segment the whole deformation dome from the acquired images and to measure its possible asymmetries which could lead to study the tissues' anisotropy. Lastly, monitoring the muscle activation might be possible using surface 
407 EMG, even though it might be difficult to avoid the electrodes placed close to LASTIC, thus 408 interfering with the aspiration device.

409

410 Acknowledgments

411 Competing interests: None.

412 Funding: This work was supported by French state funds managed by the ANR within the 413 Investissements d'Avenir programme (Labex CAMI) under reference ANR-11-LABX-0004.

414 Ethical approval: This study was conducted according to the principles expressed in the 415 Declaration of Helsinki and all participants gave their written informed consent. Ethical 416 approval was given by the CERNI committee under the reference 2013-11-19-30.

417 We would like to thank Jacques Ohayon (TIMC-IMAG) for his contribution to this work and 418 especially his help to design LASTIC and the process to estimate the stiffness modulus.

420 References

421 1. Ansys. Theory Reference. Ansys manual, release 5.6, 1999.

422 2. Ariji, Y., M. Nakayama, W. Nishiyama, and E. Ariji. Applications of sonographic 423 elastography to the oral and maxillofacial region. Journal of Radiology \& Radiation 424 Therapy, 2(2): 1049-57, 2014.

425 3. Barbarino, G.G., M. Jabareen, J. Trzewik, A. Nkengne, G. Stamatas, and E. Mazza. 426 Development and validation of a three-dimensional finite element model of the face. $\mathbf{J}$ 427 Biomech Eng, 131(4):041006-11, 2009. 
4. Barbarino G.G., M. Jabareen, and E. Mazza. Experimental and numerical study on the 429 mechanical behavior of the superficial layers of the face. Skin Res Technol. Nov;17(4):434$430 \quad 44,2011$.

5. Bickel B., P. Kaufmann, M. Skouras, B. Thomaszewski, D. Bradley, T. Beeler, P. Jackson, S. Marschner, W. Matusik, and M. Gross. Physical Face Cloning. ACM Transactions on Graphics, SIGGRAPH, vol. 31, no. 4, pp. 118:1-118:10, 2012.

6. Chabanas M., Y. Payan, C. Marécaux, P. Swider, and F. Boutault. Comparison of linear and non-linear soft tissue models with post-operative CT scan in maxillofacial surgery. Lecture Notes in Computer Science, 2004, vol. 3078, pp. 19-27.

7. Cua A. B., K. P. Wilhelm, and H. I. Maibach. Elastic properties of human skin: relation to age, sex, and anatomical region. Archives of Dermatological Research, Volume 282, Issue 5, pp 283-288, 1990.

8. Flynn C., A. Taberner, P. Nielsen, and S. Fels. Simulating the three-dimensional 441 deformation of in vivo facial skin. Journal of the Mechanical Behavior of Biomedical 442 Materials. 28, 484-494, 2013.

9. Gerard J.M., J. Ohayon, V. Luboz, P. Perrier, and Y. Payan. Non linear elastic properties of 444 the lingual and facial tissues assessed by indentation technique. Application to the 445 biomechanics of speech production, Medical Engineering \& Physics. 27 (10):884-892, 2005. 10. Hendriks F.M., D. Brokken, C.W.J. Oomens, D.L. Bader, and F.P.T. Baaijens. The 447 relative contributions of different skin layers to the mechanical behavior of human skin in 448 vivo using suction experiments. Medical Engineering \& Physics. 28: 259-266, 2006

449 11. Hollenstein M., M. Bajka, B. Röhrnbauer, S. Badir, and E. Mazza. Measuring the In Vivo 450 Behavior of Soft Tissue and Organs Using the Aspiration Device. Soft Tissue Biomechanical 
451

452

453

454

455

456

457

458

459

460

461

462

463

464

465

466

467

468

469

470

471

472

Modeling for Computer Assisted Surgery, Studies in Mechanobiology, Tissue Engineering and Biomaterials, 11:201-228, 2012.

12. Keeve E., S. Girod, R. Kikinis, and B. Girod. Deformable Modeling of Facial Tissue for Craniofacial Surgery Simulation. Journal of Computer Aided Surgery 3, 228-238,1998.

13. Kerdok A.E., M.P. Ottensmeyer, and R.D. Howe. Effects of perfusion on the viscoelastic characteristics of liver. J Biomech. 39(12):2221-31, 2006.

14. Koch R., S. Roth, M. Gross, A. Zimmermann, H. Sailer. A framework for facial surgery simulation. Technical Report 326, ETH Zurich, 1999.

15. Lapeer R.J., P.D. Gasson, and V. Karri. A hyperelastic finite-element model of human skin for interactive real-time surgical simulation. IEEE Trans Biomed Eng. 58(4):1013-22, 2011.

16. Luboz V., E. Promayon, G. Chagnon, T. Alonso, D. Favier, C. Barthod, and Y. Payan. Validation of a Light Aspiration device for in vivo Soft TIssue Characterization (LASTIC). Soft Tissue Biomechanical Modeling for Computer Assisted Surgery, Springer, 243-256, 2012.

17. Nazari M.A., P. Perrier, M. Chabanas, and Y. Payan. Shaping by stiffening: a modeling study for lips. Motor Control. Jan;15(1):141-68, 2011.

18. Ryu H.S., Y.H. Joo, S.O. Kim, K.C. Park, S.W. Youn. Influence of age and regional differences on skin elasticity as measured by the Cutometer. Skin Res Technol. 14(3):354-8, 2008.

19. Schiavone P., E. Promayon, and Y. Payan. LASTIC: a Light Aspiration device for in vivo Soft TIssue Characterization. Lecture Notes in Computer Science, 5958:1-10, 2010. 
473 20. Schiavone P., F. Chassat, T. Boudou, E. Promayon, F. Valvidia, and Y. Payan. In Vivo 474 Measurement of Human Brain Elasticity Using a Light Aspira-tion Device. Medical Image 475 Analysis, 13:673-678, 2009.

476 21. Schiavone P., T. Boudou, E. Promayon, P. Perrier \& Y. Payan. A light sterilizable pipette 477 device for the in vivo estimation of human soft tissues constitutive laws. Proceedings of the 478 30th Annual International Conference of the IEEE Engineering in Medicine and Biology 479 Society, IEEE EMBS 2008, 4298-4301, 2008.

480 22. Zachow S., E. Gladiline, H. Hege, and P. Deuflhard. Finite element simulation for soft 481 tissue prediction. In Lemke, H., al., eds.: Computer Assisted Radiology and Surgery, 482 CARS'00, Elsevier 23-28, 2000.

483 23. Welch B. L. The generalization of "Student's" problem when several different population 484 variances are involved. Biometrika 34 (1-2): 28-35, 1947. 
Figures and tables

486

487 Figure 1 Luboz

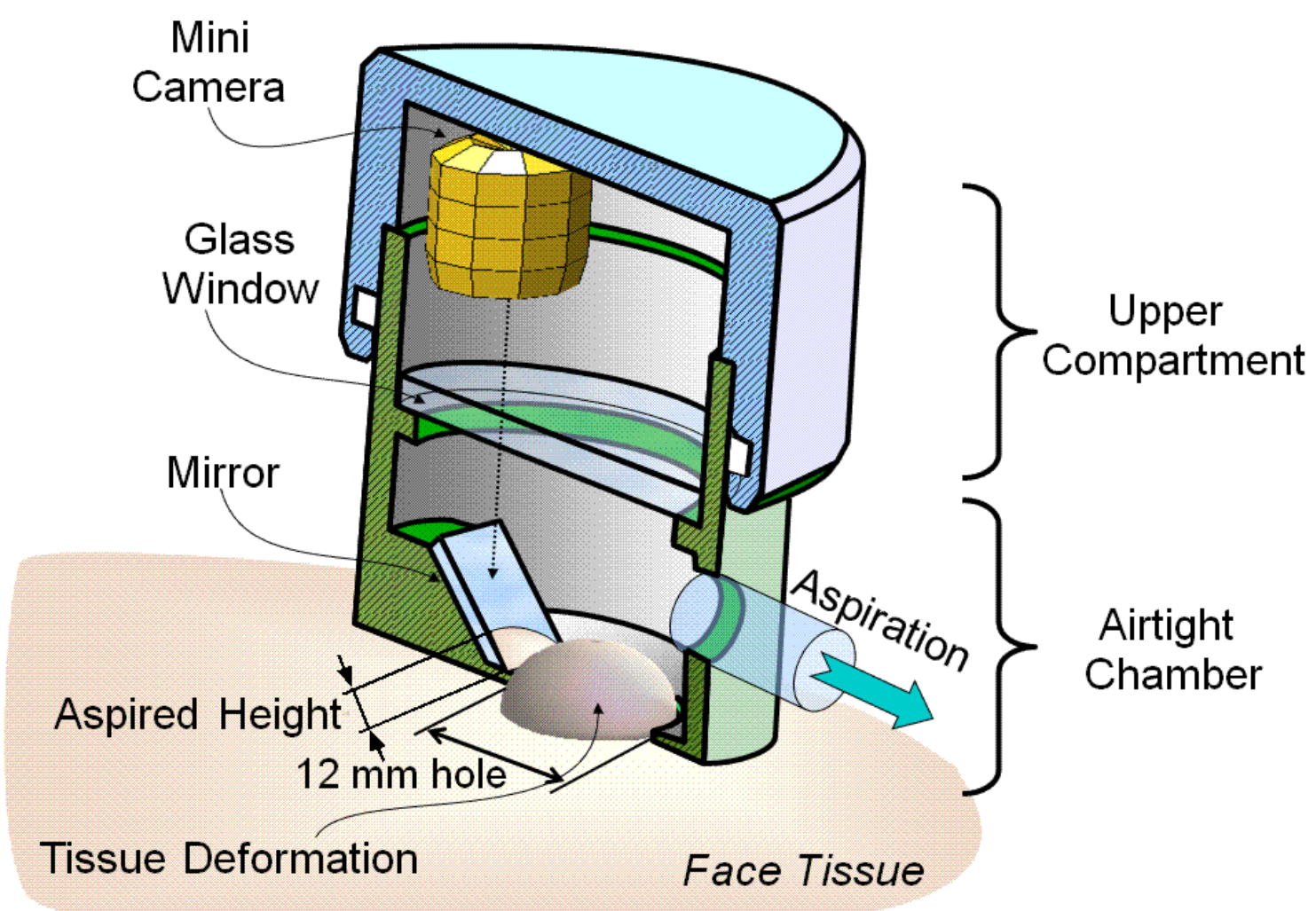


489 Figure 2 Luboz

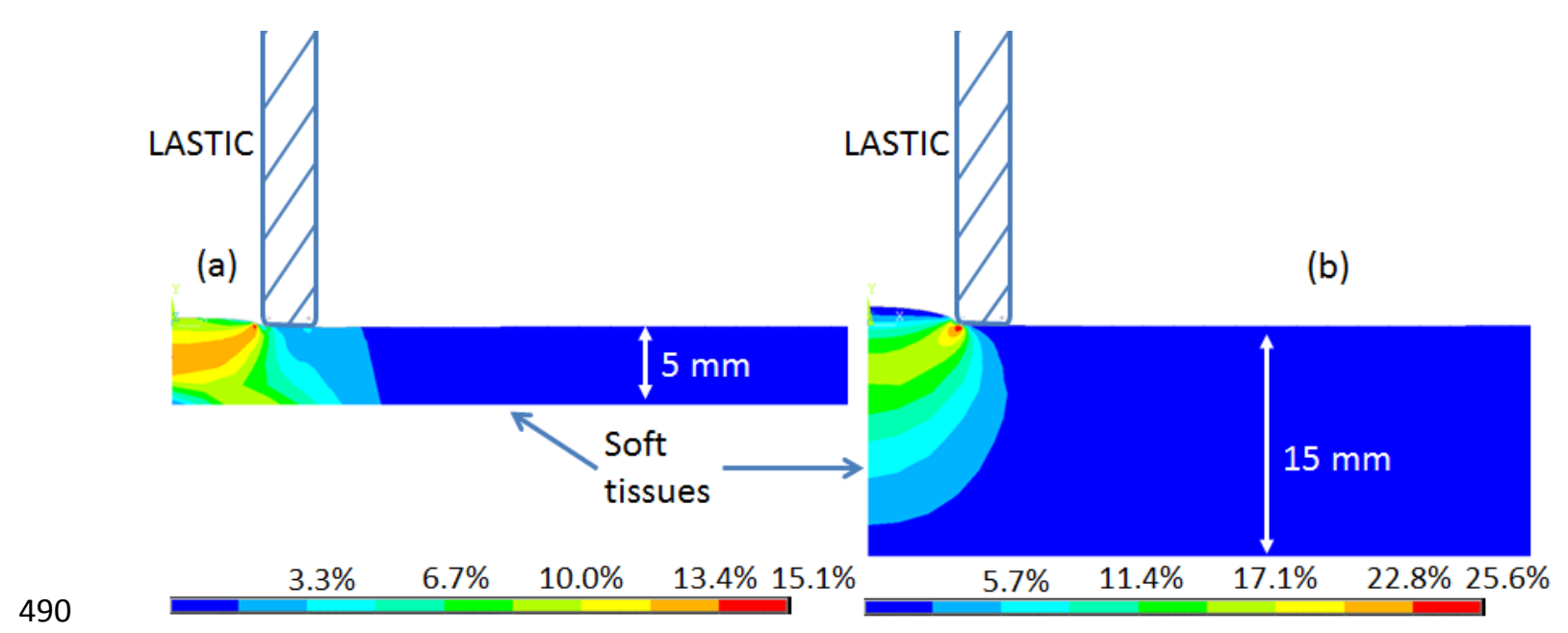

491 


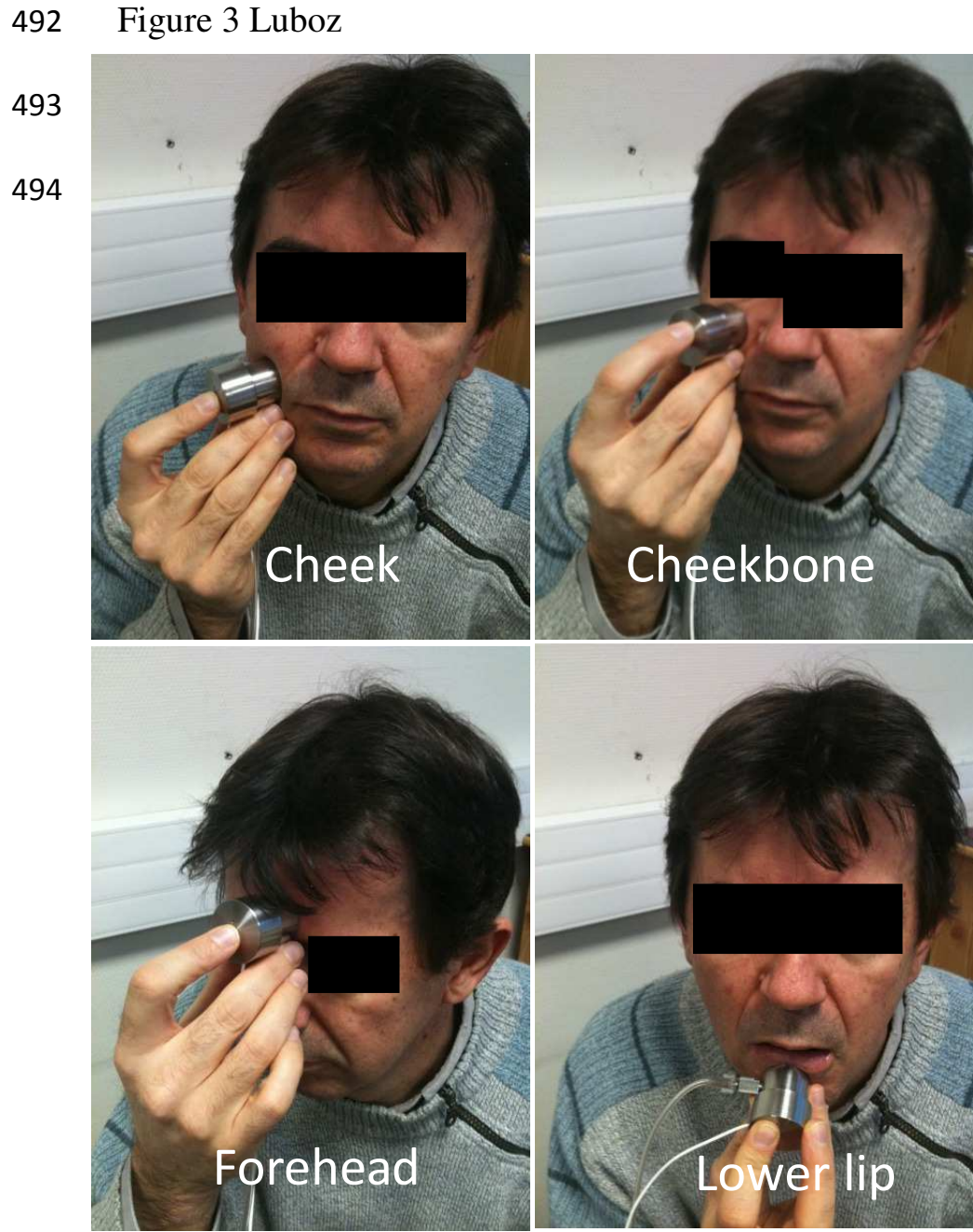




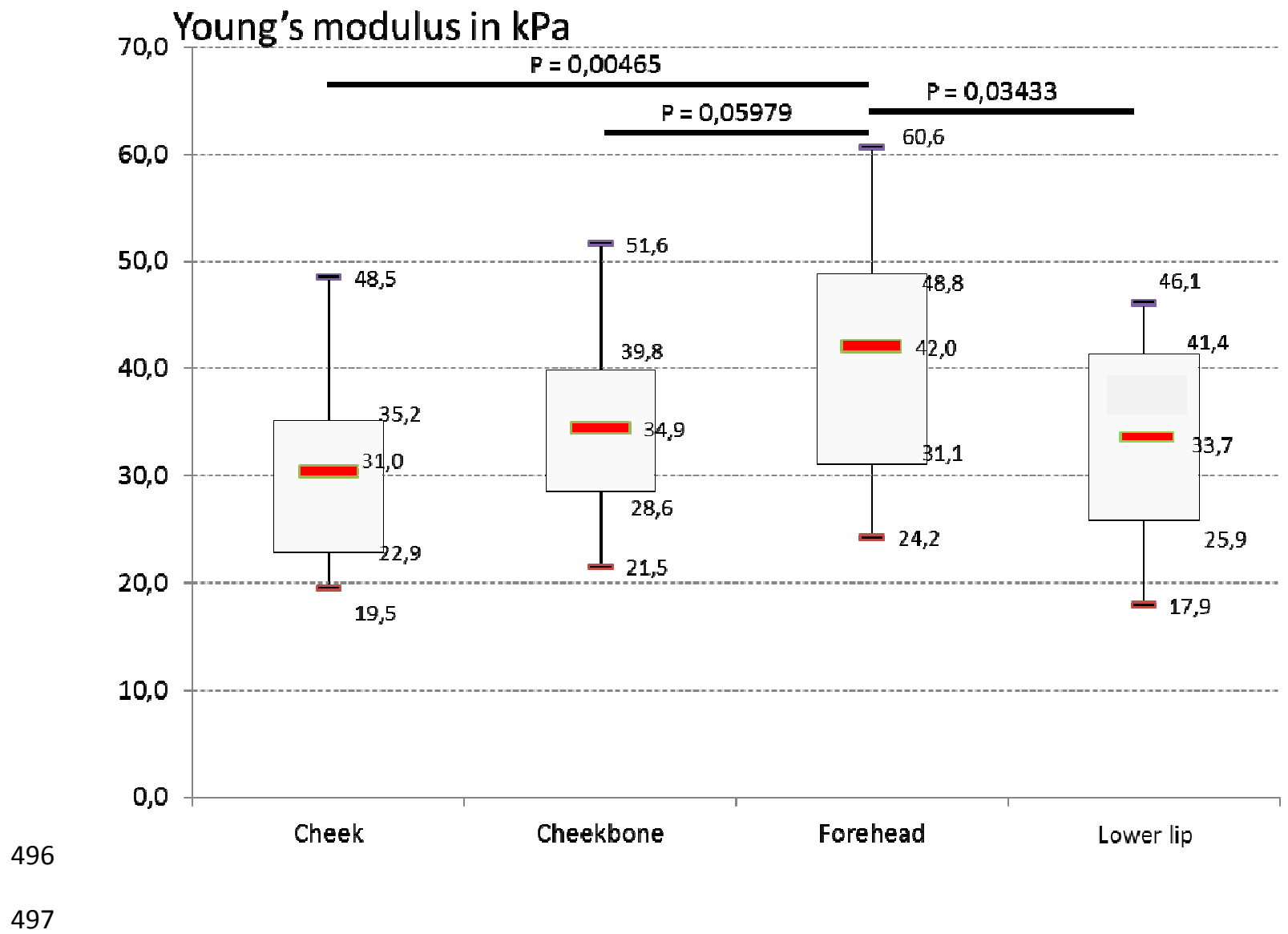


Figure 5 Luboz

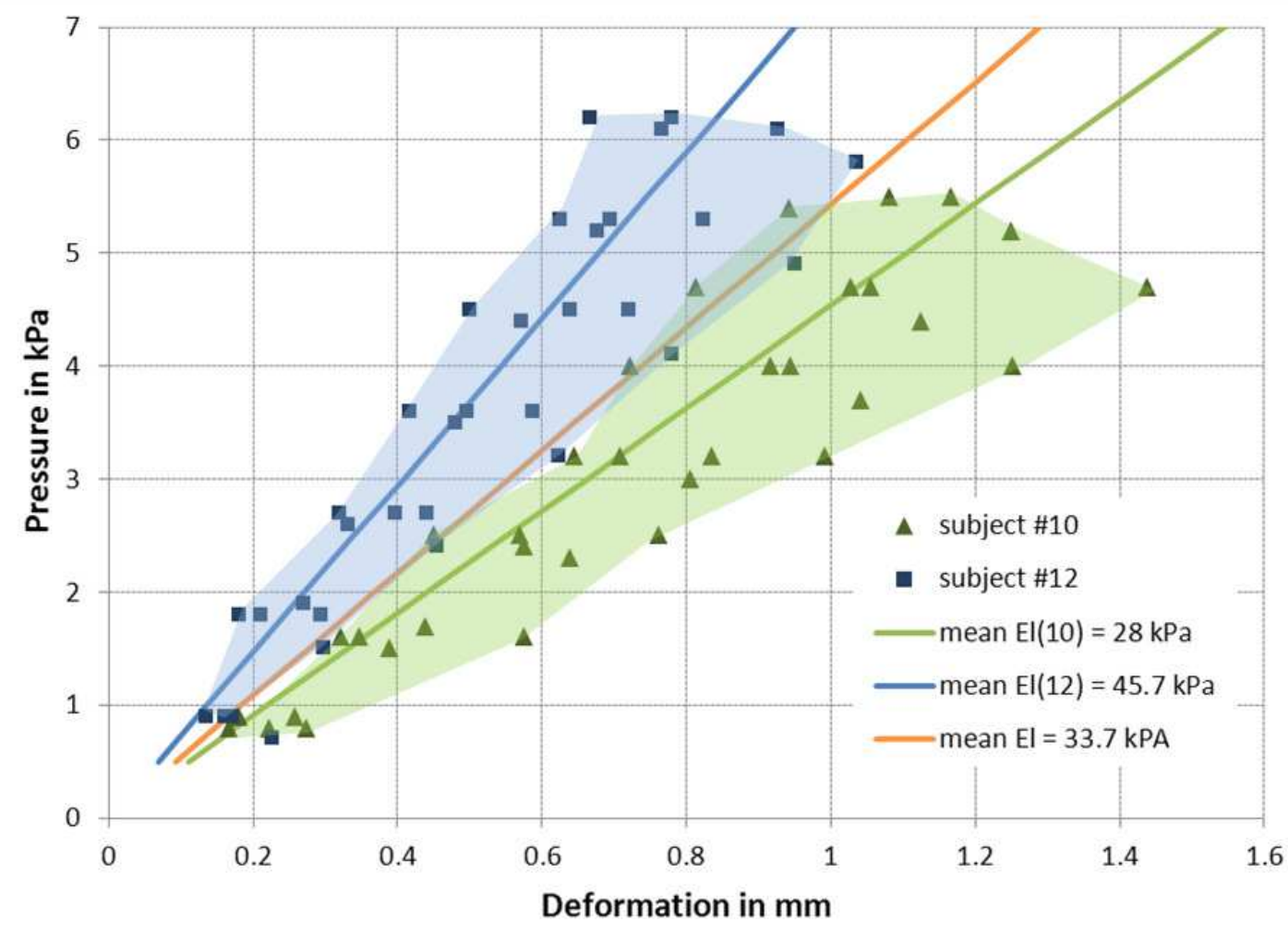

499 
Table 1 Luboz

\begin{tabular}{|c|c|c|c|c|c|c|}
\hline Subjec & & & & Cheekbone & & \\
\hline $\mathrm{t} \#$ / & age & BMI (kg.m ${ }^{-}$ & Cheek $E_{c}(i)$ & $\mathrm{E}_{\mathrm{cb}}(\mathrm{i}) \pm \mathrm{std}$ & Forehead $\mathrm{E}_{\mathrm{f}}(\mathrm{i})$ & Lower lip $\mathrm{E}_{1}(\mathrm{i})$ \\
\hline $\operatorname{sex}$ & (years) & 2 ) & $\pm \operatorname{std}(\mathrm{kPa})$ & $(\mathrm{kPa})$ & $\pm \operatorname{std}(\mathrm{kPa})$ & $\pm \operatorname{std}(\mathrm{kPa})$ \\
\hline $1 / \mathrm{M}$ & 44 & 22.0 & $21.3 \pm 3.7$ & $21.5 \pm 2.1$ & $10.6 \pm 1.6$ & $19.1 \pm 3.4$ \\
\hline $2 / \mathrm{M}$ & 35 & 21.2 & $20.7 \pm 4.5$ & $23.1 \pm 3.4$ & $9.9 \pm 2.4$ & $20.1 \pm 4.1$ \\
\hline $3 / \mathrm{F}$ & 26 & 20.7 & $23.4 \pm 7.4$ & $29.0 \pm 11.8$ & $16.0 \pm 2.7$ & $46.1 \pm 12.7$ \\
\hline $4 / \mathrm{F}$ & 35 & 22.8 & $48.5 \pm 9.9$ & $50.7 \pm 5.0$ & $24.2 \pm 7.4$ & $41.2 \pm 7.2$ \\
\hline $5 / \mathrm{F}$ & 26 & 19.2 & $21.1 \pm 5.0$ & $22.1 \pm 2.5$ & $10.5 \pm 2.9$ & $33.3 \pm 8.2$ \\
\hline $6 / \mathrm{M}$ & 23 & 21.1 & $28.9 \pm 3.3$ & $32.1 \pm 2.8$ & $13.0 \pm 2.9$ & $27.1 \pm 7.2$ \\
\hline $7 / \mathrm{F}$ & 24 & 20.3 & $34.5 \pm 4.6$ & $39.3 \pm 13.8$ & $30.2 \pm 7.9$ & $36.5 \pm 7.9$ \\
\hline $8 / \mathrm{M}$ & 23 & 21.9 & $19.5 \pm 2.3$ & $27.3 \pm 6.6$ & $11.7 \pm 3.5$ & $17.9 \pm 4.6$ \\
\hline $9 / \mathrm{M}$ & 39 & 26.0 & $31.2 \pm 4.0$ & $41.4 \pm 5.8$ & $18.9 \pm 3.8$ & $39.8 \pm 11.4$ \\
\hline $10 / F$ & 24 & 16.8 & $37.1 \pm 4.9$ & $39.3 \pm 10.9$ & $19.5 \pm 5.1$ & $28.0 \pm 5.9$ \\
\hline $11 / \mathrm{M}$ & 27 & 25.1 & $29.1 \pm 3.3$ & $39.1 \pm 10.1$ & $22.8 \pm 1.8$ & $38.0 \pm 11.9$ \\
\hline $12 / \mathrm{M}$ & 27 & 19.9 & $31.6 \pm 4.0$ & $30.5 \pm 8.1$ & $24.8 \pm 6.6$ & $45.7 \pm 7.9$ \\
\hline $13 / \mathrm{M}$ & 27 & 22.1 & $43.1 \pm \pm 2.6$ & $51.6 \pm \pm 7.5$ & $15.9 \pm 4.1$ & $42 \pm 12.8$ \\
\hline $14 / F$ & 32 & 21.5 & $44.5 \pm 3.7$ & $42.4 \pm 3.7$ & $21.9 \pm 7.5$ & $39.1 \pm 3.8$ \\
\hline $15 / F$ & 32 & 21.2 & $29.5 \pm 3.3$ & $35.1 \pm 1.0$ & $15.6 \pm 2.9$ & $22.3 \pm 1.0$ \\
\hline $16 / F$ & 24 & 22.3 & $32.4 \pm 7.0$ & $33.7 \pm 11.0$ & $11.5 \pm 2.9$ & $43.4 \pm 6.3$ \\
\hline Mean & $29.3 \pm 6$ & & $\mathrm{E}_{\mathrm{c}}=$ & $\mathrm{E}_{\mathrm{cb}}=$ & & \\
\hline \pm std & 3 & $21.5 \pm 2.1$ & $31.0 \pm 4.6$ & $34.9 \pm 6.6$ & $\mathrm{E}_{\mathrm{f}}=17.3 \pm 4.1$ & $E_{1}=33.7 \pm 7.3$ \\
\hline
\end{tabular}




\begin{tabular}{|l|r|r|r|}
\hline & \multicolumn{1}{|l|}{ Cheek $\mathrm{E}_{\mathrm{c}}$} & Lower lip $\mathrm{E}_{\mathrm{l}}$ & Cheekbone $\mathrm{E}_{\mathrm{cb}}$ \\
\hline Forehead $\mathrm{E}_{\mathrm{f}}$ & 0.000025 & 0.000007 & 0.000001 \\
\hline Cheek $\mathrm{E}_{\mathrm{c}}$ & & 0.424 & 0.238 \\
\hline Lower lip $\mathrm{E}_{\mathrm{l}}$ & & & 0.733 \\
\hline
\end{tabular}


Captions:

503 Figure 1 - Cross section of LASTIC's two compartments. The lower part is the aspiration 504 chamber with the mirror and the upper part contains the camera that images the deformation. $505 \quad$ Figure 2 - The two different Finite Element models used to create the precomputed library 506 of displacement heights / pressure curves, for a tissue thickness of (a) $5 \mathrm{~mm}$ (forehead), and 507 (b) $15 \mathrm{~mm}$ or more (lip, cheek, cheekbone). The Von Mises strains are plotted on both sides, 508 showing maximum deformations (of (a) $15 \%$ and (b) $26 \%$ ) near the interface with LASTIC 509 because of the contact. But the measured displacement height is at the top of the deformation 510 dome.

$511 \quad$ Figure 3 - The measurements are done at four locations: the cheek, the cheekbone, the 512 forehead, and the lower lip. The subjects are asked to position and hold LASTIC themselves 513 under supervision of the experimenter. From the LASTIC measurements, the stiffness of the 514 facial soft tissues at the different location can be estimated.

$515 \quad$ Figure 4 - Whisker box plot showing for each location: mean, minimal and maximal initial 516 Young's modulus (in $\mathrm{kPa}$ ). The result of the bilateral paired Welch's T-test between the 517 forehead and the three other locations is also presented.

518 Figure 5 - Variation of the initial Young's modulus $E_{l}$ (lower lip) for subjects \#10 and \#12 519 (averaged for the five measurements), compared to the mean initial Young's modulus for all 520 subjects. The mean stiffness computed by the inverse analysis using a Neo Hookean 521 approximation is also plotted (continuous lines). Measurement points are also plotted. $E_{l}(10)$ $522=E_{l} \times 0.83$ and $E_{l}(12)=E_{l} \times 1.35$. 
523 Table 1 - Age, body mass index (BMI), sex (M for Male and F for Female), initial Young's 524 modulus $E$ (in $\mathrm{kPa}$ ) and standard deviation (std) of each subject $i$ for the facial soft tissues at 525 four locations: cheek $\left(E_{c}\right)$, cheekbone $\left(E_{c b}\right)$, forehead $\left(E_{f}\right)$, and lower lip $\left(E_{l}\right)$.

526 Table 2 - P-value for each possible pair of locations where the stiffness is estimated.

527

528

529 